\section{Effects of Drying and Extraction Conditions on the Biochemical Activity of Selected Herbs}

\author{
Weiguang Yi and Hazel Y. Wetzstein ${ }^{1}$ \\ Department of Horticulture, 1111 Plant Science Building, The University of \\ Georgia, Athens, GA 30602-7273
}

Additional index words. antioxidant capacity, motherwort, peppermint, phenolics, rosemary, TEAC

\begin{abstract}
Herbs have been long known to provide health-promoting benefits and are demonstrated to have antioxidant, anti-inflammatory, antibacterial, analgesic, and antitumor activities. This study evaluated the effects of drying conditions and extraction protocols on the biochemical activity of three culinary and medicinal herbs: rosemary (Rosmarinus officinalis), motherwort (Leonurus cardiaca), and peppermint (Mentha piperita). Leaf tissues were dried by sun, oven-dried at $40{ }^{\circ} \mathrm{C}$, or oven-dried at $70{ }^{\circ} \mathrm{C}$ and extracted using $80 \%$ methanol or $80 \%$ ethanol. Total polyphenol (TPP) using the FolinCiocalteu reagent method and antioxidant capacity using the Trolox-equivalent antioxidant capacity (TEAC) assay were determined. Both drying and extraction conditions significantly impacted TPP content and TEAC in the three herb species. Sun-dried or $40{ }^{\circ} \mathrm{C}$ oven-dried herbs exhibited significantly higher TPP content and TEAC capacity than fresh samples, suggesting low-temperature drying may be a good postharvest means to store medicinal/culinary herbs. Exposure to $70^{\circ} \mathrm{C}$ oven-drying caused significant antioxidant loss. In addition, the current study showed that with fresh tissue, $80 \%$ ethanol extraction had significantly higher TPP and TEAC than $80 \%$ methanol extraction for all three herbs, yet for dried herbs, the efficacy of ethanol/methanol extraction varied with different drying treatments.
\end{abstract}

Herbs and spices have been used not only as food preservatives and flavoring, but also as traditional medicines for thousands of years. Two-thirds of the world's population still relies on traditional medicines with herbal medicines the most common form. The World Health Organization (2010) estimates that $80 \%$ of the population in some Asian and African countries depends on traditional medicines for primary health care. In the United States, the Centers for Disease Control and Prevention estimates $\approx 38 \%$ of adults use complementary and alternative medical treatments. Scientific evidence increasingly supports the potential health benefits of herbs with plant extracts displaying antioxidant, antiinflammatory, antibacterial, analgesic, and antitumor activities.

Oxygen-free radical induced oxidation of cellular components is believed to be one of the major factors in the development of heart disease and cancer (Berger, 2005). Oxidizing agents, often in the form of free radical compounds, can cause damage to numerous cellular components, including membranes, proteins, and nucleic acids (Knight, 2000). Dietary intake of herbs and spices with high antioxidant contents may improve our resistance to free radical damage and thus reduce risk of heart disease and cancer (Kaefer and Milner, 2008).

Received for publication 16 Aug. 2010. Accepted for publication 4 Nov. 2010.

${ }^{1}$ To whom reprint requests should be addressed; e-mail hywetz@uga.edu.
Studies show that herbs generally contain higher levels of antioxidant content than fruits, vegetables, and nuts (Konczak et al., 2010; Zheng and Wang, 2001). More and more people have realized the health benefits of herbs and the use of herbs is increasing (Kaefer and Milner, 2008).

The drying of spices has been used for disinfestations, microbial decontamination, and long-term preservation (Schweiggert et al., 2007). Although reports are limited, studies indicate that drying conditions can impact the chemical and biological activities of herbs (Capecka et al., 2005; Lim and Murtijaya, 2007). The Mint family, Lamiaceae, is a group of $\approx 210$ genera and some 3500 species. Many members of the family have high phenolic and antioxidant content such as basil (Ocimum basilicum), lemon balm (Melissa officinalis), sweet marjoram (Origanum majorana), oregano (Origanum vulgare), peppermint, rosemary, sage (Salvia officinalis), and thyme (Thymus vulgaris) (Dragland et al., 2003; Yi and Wetzstein, 2010). Systematic evaluations of drying and extraction conditions on biochemical activities of this group are lacking. The objectives of this study were to evaluate the effects of drying conditions and extraction protocols on total phenolic content and antioxidant capacity of selected herbs.

\section{Materials and Methods}

Plant materials, harvesting, and drying. Three herbs, rosemary (Rosmarinus officinalis), motherwort (Leonurus cardiaca), and peppermint (Mentha piperita), were grown in a greenhouse at the University of Georgia, Athens, GA, from Feb. 2009 to Apr. 2009. Herbs were planted in $30-\mathrm{cm}$ wide pots with Fafard 3B soil and grown under natural light conditions at $21 \pm 2{ }^{\circ} \mathrm{C}$. The plant heights at harvest were: peppermint, 35 to $40 \mathrm{~cm}$; motherwort, 45 to $55 \mathrm{~cm}$; and rosemary, 40 to $45 \mathrm{~cm}$. For all species, tissue samples were collected from the apical portion of branches and consisted of the youngest, fully expanded leaves. None of the plants were flowering. Average leaf sizes were: peppermint, $1.7 \mathrm{~cm}$ wide $\times 2.7 \mathrm{~cm}$ long; motherwort (collected without petioles), $7.0 \mathrm{~cm}$ wide $\times 7.2 \mathrm{~cm}$ long; and rosemary, $0.34 \mathrm{~cm}$ wide $\times 3.2 \mathrm{~cm}$ long.

Freshly harvested leaf tissue was subjected to one of three drying methods: 1) sun-dried in the greenhouse; 2) oven-dried at $40{ }^{\circ} \mathrm{C}$ in a forced-air oven; or 3 ) oven-dried at $70{ }^{\circ} \mathrm{C}$ in a forced-air oven. Tissues were dried for $4 \mathrm{~d}$. The moisture content of the tissues was calculated using a subset of tissues dried under $70{ }^{\circ} \mathrm{C}$ in a ventilation oven. Fresh samples served as a control.

Chemicals. Pure standards of gallic acid, 2,2' -azinobis(3-ethylbenzothiazoline-6-sulfonic acid) diammonium salt (ABTS), Trolox (6-hydroxy-2,5,7,8-tetramethychroman2 -carboxylic acid), and Folin-Ciocalteu reagent were purchased from Sigma (St. Louis, MO). Ethanol [high-performance liquid chromatography (HPLC) grade], methanol (HPLC grade), and potassium persulfate were purchased from Fisher Scientific (Norcross, GA).

Extraction. Extraction procedures followed a previous report of Yi and Wetzstein (2010). Fresh leaf samples, sun-dried samples, $40{ }^{\circ} \mathrm{C}$ oven-dried, or $70{ }^{\circ} \mathrm{C}$ oven-dried samples (each replication was on $3 \mathrm{~g}$ of a fresh weight basis) were homogenized with a frozen mortar and pestle in liquid nitrogen. Tissues were extracted for $2 \mathrm{~h}$ at room temperature under darkness in $50 \mathrm{~mL}$ of $80 \%$ methanol or $80 \%$ ethanol with agitation on an orbital shaker at $200 \mathrm{rpm}$. The crude extract solutions were centrifuged at $8000 \mathrm{~g}$ for $15 \mathrm{~min}$ and supernatants were collected. Tissues were reextracted for $1 \mathrm{~h}$ in the same solvent for two more times. The extracts were combined and then further passed through $0.2-\mu \mathrm{m}$ nylon filters.

Total polyphenol measurement. TPP was measured according to the Folin-Ciocalteu reagent method (Singleton and Rossi, 1965). Sample solutions were mixed with FolinCiocalteu reagent and 7.5\% sodium carbonate $(\mathrm{w} / \mathrm{v})$ and allowed to stand for $30 \mathrm{~min}$. Absorption at $765 \mathrm{~nm}$ was measured using a spectrophotometer (Spectronic 21D; Milton Roy, Rochester, NY). The total phenolic content was expressed as milligrams of gallic acid equivalent per gram of dry extract (GAE $\mathrm{mg} \cdot \mathrm{g}^{-1}$ ) using a standard curve generated with $50,100,200,300$, and $400 \mathrm{mg} \cdot \mathrm{L}^{-1}$ of gallic acid.

Antioxidant capacity measurement. The antioxidant capacity was measured following a slight modification of the method reported by Re et al. (1999). In the modified procedure, $30 \mu \mathrm{L}$ of extract was added to $2970 \mu \mathrm{L}$ of 
ABTS (Yi and Wetzstein, 2010). For calibration, Trolox (6-hydroxy-2,5,7,8-tetramethychroman-2-carboxylic acid, a vitamin $\mathrm{E}$ analog) standards of $0,300,600,900,1200$, and 1500 $\mu \mathrm{M}$ were used. The TEAC of the sample was calculated based on the inhibition exerted by the standard Trolox solution at $6 \mathrm{~min}$. Absorption at $734 \mathrm{~nm}$ was measured using a spectrophotometer (Spectronic 21D; Milton Roy). TEAC antioxidant capacity was expressed as TE $\mu \mathrm{M} \cdot \mathrm{g}^{-1}$, which stands for micromolar of Trolox equivalent per gram of dry extract.

Statistical analysis. Statistical analysis was conducted using the General Linear Model (SAS 9.1; SAS Inst., Inc., Cary, NC). The TPP content, TEAC antioxidant capacity of three selected herbs under different drying, and extraction conditions were assessed by Student's $t$ test and compared by Duncan's multiple range test at $\alpha=0.05$.

\section{Results}

The TPP content and TEAC antioxidant capacity of rosemary under different extraction and processing conditions are shown in Figure 1A-B. Among the four different drying conditions, the highest TPP value was observed with sun-dried leaves. The TPP was 39.6 GAE $\mathrm{mg} \cdot \mathrm{g}^{-1}$ when extraction was conducted using methanol, and $34.3 \mathrm{GAE} \mathrm{mg} \cdot \mathrm{g}^{-1}$ when extraction was conducted using ethaoven-drying at 40 or $70{ }^{\circ} \mathrm{C}$ resulted in similar TPP values; with ethanol extraction, ovendrying at 40 and $70{ }^{\circ} \mathrm{C}$ resulted in the lowest TPP values. TPP was significantly highest with nol. With methanol extraction, fresh, and

sun-drying, even beyond that in fresh tissue. Oven-drying at either temperature resulted in TPP concentrations similar to fresh tissues when extracted with methanol or significantly lower than fresh or sun-dried tissues with ethanol extractions.

Similar results were obtained in TEAC antioxidant capacity of rosemary (Fig. 1B). For the methanol extraction, the highest TEAC value (TE $291.8 \mu \mathrm{M} \cdot \mathrm{g}^{-1}$ ) was observed with sun-drying; fresh, oven-drying at 40 or $70{ }^{\circ} \mathrm{C}$ resulted in significantly lower TEAC values than sun-drying. With ethanol extraction, fresh and sun-drying showed significantly higher TEAC values than oven-drying at 40 and $70{ }^{\circ} \mathrm{C}$. The type of solvent used during extraction had a significant effect in fresh and sun-dried samples but not in oven-dried tissues. With fresh tissue, significantly higher TPP and TEAC were obtained with ethanol versus methanol extractions. In contrast, with sun-dried samples, higher TPP and TEAC values were obtained using methanol versus ethanol.

In contrast to rosemary, motherwort consistently had the highest TPP and TEAC values with tissues that were dried at $40{ }^{\circ} \mathrm{C}$ (Fig. 2A-B). When Leonurus leaves were extracted using methanol, $40{ }^{\circ} \mathrm{C}$ oven drying resulted in the highest TPP (GAE $38.1 \mathrm{mg} \cdot \mathrm{g}^{-1}$ ) compared with fresh samples, which showed the lowest TPP at GAE $17.5 \mathrm{mg} \cdot \mathrm{g}^{-1}$. Likewise, when extraction was conducted using ethanol, the highest TPP was observed with $40{ }^{\circ} \mathrm{C}$ oven-dried samples. Successively lower values were obtained in sun-dried, $70^{\circ} \mathrm{C}$ ovendried, and fresh tissues. With ethanol extrac-
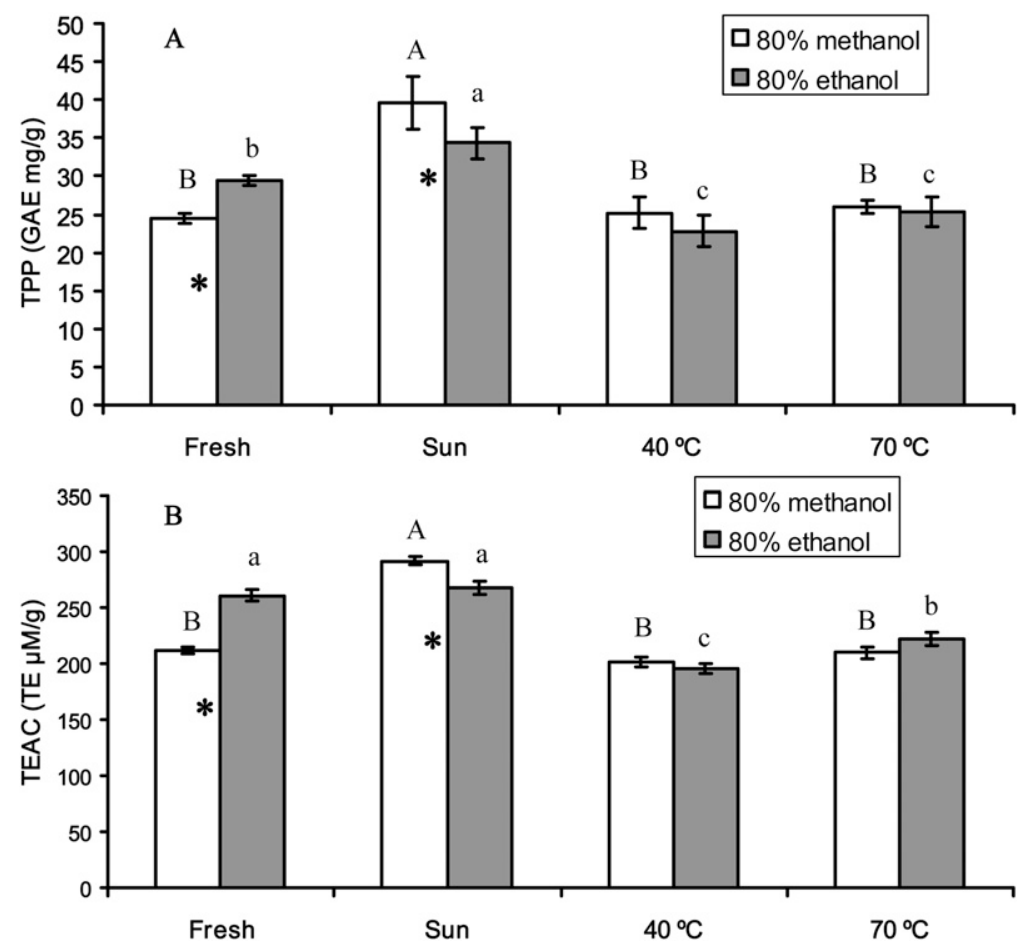

Fig. 1. The total polyphenol contents (A) and Trolox-equivalent antioxidant capacity antioxidant capacity (B) of rosemary leaves under different drying and extraction conditions. Mean separations among drying conditions within an extraction solvent are shown. Values followed by different letters (upper case for $80 \%$ methanol, lower case for $80 \%$ ethanol) are significantly different at $\alpha=0.05$. $*$ Significant difference (at $\alpha=0.05$ ) between methanol versus ethanol extraction using the same drying method. tion, TPP decreased by $27 \%, 58 \%$, and $64 \%$, respectively. Although methanol extractions followed similar trends, differences were somewhat smaller.

Similar results were obtained in the TEAC antioxidant capacity of Leonurus leaves with the highest values likewise obtained with drying at $40{ }^{\circ} \mathrm{C}$ (Fig. 2B). With methanol extraction, the highest TEAC value (TE 297.8 $\mu \mathrm{M} \cdot \mathrm{g}^{-1}$ ) observed with $40{ }^{\circ} \mathrm{C}$ oven-drying dropped by $6 \%$ with sun-drying, $36 \%$ with $70{ }^{\circ} \mathrm{C}$ drying, and $78 \%$ in fresh samples. In the case of ethanol extractions, similar trends were obtained with the highest TEAC value found with $40{ }^{\circ} \mathrm{C}$ drying and the lowest in fresh samples. The type of solvent used in extraction can have a significant effect on TPP and TEAC depending on the method of drying. In the case of TPP, ethanol resulted in significantly higher values in fresh, sundried, and $40{ }^{\circ} \mathrm{C}$ dried tissues than methanol extraction. TEAC activity was also higher with ethanol extractions, but only in fresh and $40{ }^{\circ} \mathrm{C}$ drying treatments.

The TPP content and TEAC antioxidant capacity of peppermint leaves under different processing conditions are shown in Figure $3 \mathrm{~A}-\mathrm{B}$. In general, the highest TPP and TEAC values were observed in tissues that were either sun-dried or oven-dried at $40{ }^{\circ} \mathrm{C}$. Highest TPP (GAE $86.0 \mathrm{mg} \cdot \mathrm{g}^{-1}$ ) and TEAC (TE $366.8 \mu \mathrm{M} \cdot \mathrm{g}^{-1}$ ) values were obtained with methanol extraction in tissues dried at $40{ }^{\circ} \mathrm{C}$. Similar to what was observed in motherwort, successively lower values were obtained in sun-dried, $70{ }^{\circ} \mathrm{C}$ oven-dried, and fresh tissues. With ethanol extractions, TPP in sundried and $40^{\circ} \mathrm{C}$ dried tissues were similar and significantly higher that fresh and $70{ }^{\circ} \mathrm{C}$ dried samples. The highest TEAC value (TE 382.5 $\mu \mathrm{M} \cdot \mathrm{g}^{-1}$ ) was observed with sun-dried tissues followed by $40{ }^{\circ} \mathrm{C}, 70{ }^{\circ} \mathrm{C}$, and fresh samples. The effect of solvent extraction on TPP and TEAC values depended on the method of drying. In both TPP and TEAC, ethanol resulted in significantly higher values in fresh and sun-dried tissues than methanol. However, ethanol was less effective than methanol if tissue was dried at $40{ }^{\circ} \mathrm{C}$.

The ranking order of TEAC values of peppermint leaves among different postharvest treatments varied with the solvent. When the extraction was conducted using methanol, $40{ }^{\circ} \mathrm{C}$ oven-dried samples showed the highest TEAC value followed by sun-drying, $70{ }^{\circ} \mathrm{C}$ oven-drying, and then fresh samples. Meanwhile, when the extraction was conducted using ethanol, sun-dried samples showed the highest TEAC value (TE $63.8 \mu \mathrm{M} \cdot \mathrm{g}^{-1}$ ) followed by oven-drying at $40{ }^{\circ} \mathrm{C}, 70{ }^{\circ} \mathrm{C}$, and then fresh samples. When comparing different solvents, ethanol gave significantly higher TPP and TEAC in fresh and sun-dried peppermint leaves than methanol, although methanol resulted in higher TPP and TEAC in $40{ }^{\circ} \mathrm{C}$ oven-dried samples.

\section{Discussion}

Heat processing can have detrimental effects on natural antioxidants in raw plant 

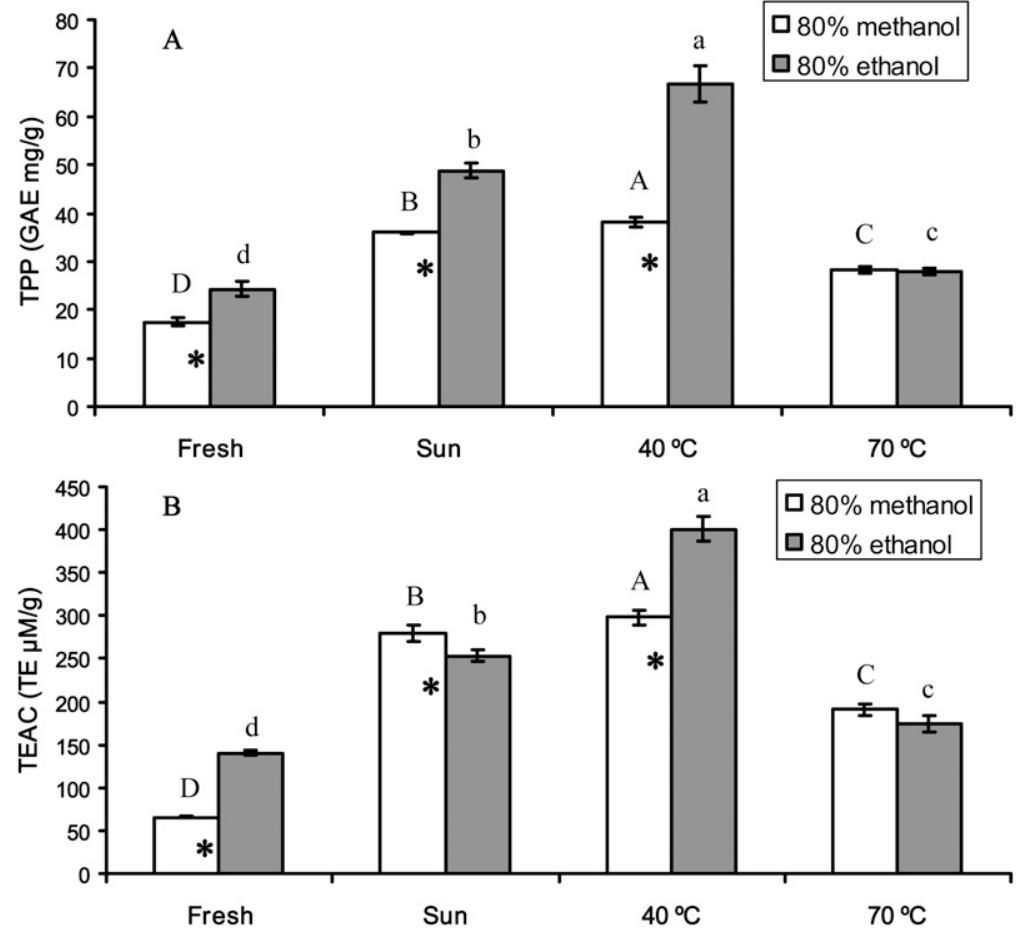

Fig. 2. The total polyphenol contents (A) and Trolox-equivalent antioxidant capacity antioxidant capacity (B) of motherwort leaves under different drying and extraction conditions. Mean separations among drying conditions within an extraction solvent are shown. Values followed by different letters (upper case for $80 \%$ methanol, lower case for $80 \%$ ethanol) are significantly different at $\alpha=0.05$. * Significant difference (at $\alpha=0.05$ ) between methanol versus ethanol extraction using the same drying method.
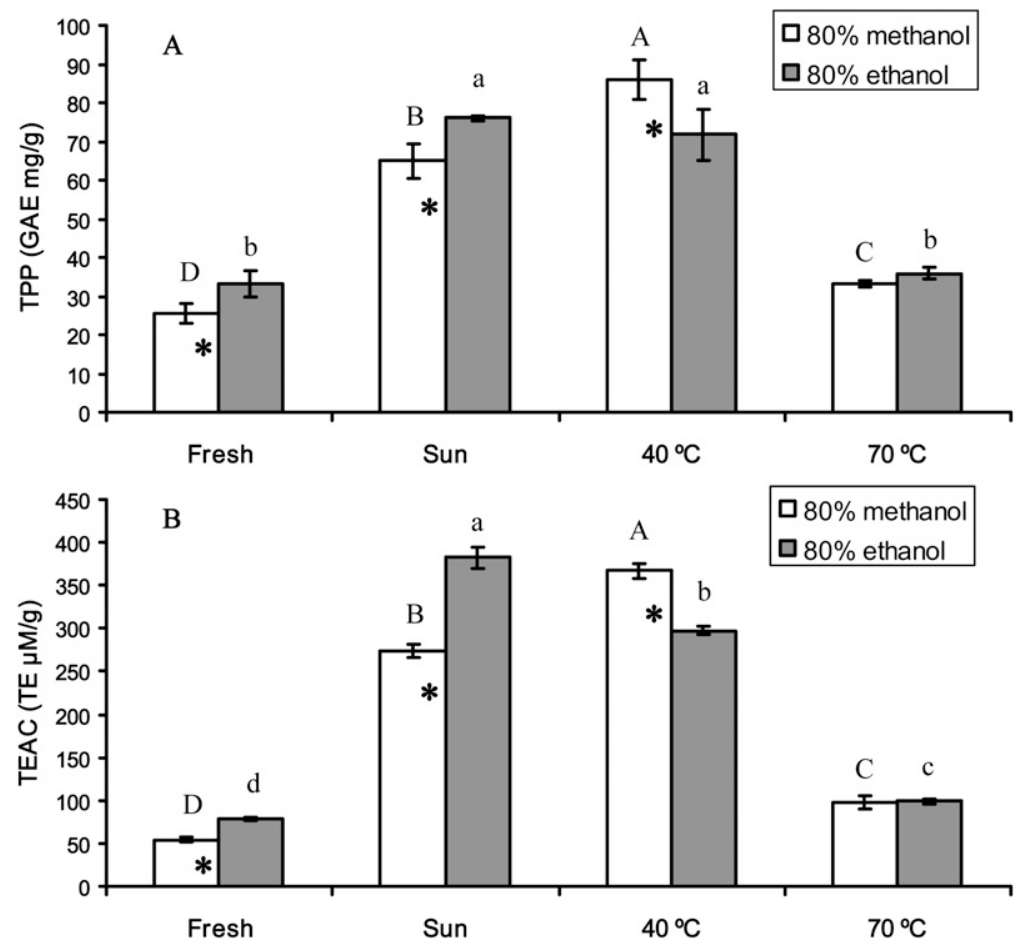

Fig. 3. The total polyphenol contents (A) and Trolox-equivalent antioxidant capacity antioxidant capacity (B) of peppermint leaves under different drying and extraction conditions. Mean separations among drying conditions within an extraction solvent are shown. Values followed by different letters (upper case for $80 \%$ methanol, lower case for $80 \%$ ethanol) are significantly different at $\alpha=0.05$. * Significant difference (at $\alpha=0.05$ ) between methanol versus ethanol extraction using the same drying method.

materials. Drying of sea buckthorn (Hippophae rhamnoides) leaves at temperatures ranging from 50 to $100{ }^{\circ} \mathrm{C}$ resulted in de- creased concentrations of TPP with greater reductions observed with higher temperatures and longer times (Guan et al., 2005).
In the herb, Phyllanthus amarus, oven- or microwave-drying treatments led to significant reductions in antioxidant property with microwave-drying causing the highest decrease in total polyphenols, radical scavenging activity, and ferric-reducing antioxidant power antioxidant capacity (Lim and Murtijaya, 2007). Intense and/or prolonged thermal treatment can cause significant loss as a result of the heat instability of compounds (Tomaino et al., 2005). In the current study, the significant loss of TPP and TEAC at $70^{\circ} \mathrm{C}$ compared with sun- or $40{ }^{\circ} \mathrm{C}$ oven-drying was likely the result of the breakdown of phenolic compounds under high heat conditions.

In contrast to high temperature, the current study showed drying at low temperature can increase total polyphenol contents and antioxidant capacity. Sun-drying and $40{ }^{\circ} \mathrm{C}$ oven-drying resulted in significantly higher TPP and TEAC than fresh samples of peppermint and motherwort. It is not uncommon that drying can increase TPP and antioxidant capacity. Oboh and Akindahunsi (2004) reported that sun-drying caused a significant increase in TPP, reducing property, and free radical scavenging ability of green leafy vegetables, although it can cause significant loss of vitamin C. Capecka et al. (2005) reported that air-drying of oregano and peppermint at 25 to $32{ }^{\circ} \mathrm{C}$ resulted in significant increases in TPP. This agrees with our current study, which generally showed sun and $40{ }^{\circ} \mathrm{C}$ oven-drying resulted in significant increases to TPP and TEAC in herbs.

The increase of phenolic contents and antioxidant capacity may be the result of the formation of novel compounds with antioxidant activity. Tomaino et al. (2005) suggest that the increase of safrole, myristicin, and other secondary compounds could be associated with increased free radical scavenging activity as a result of heating in nutmeg (Myristica fragrans). Similar bioconversion phenomena were reported in Artemisia annua leaves (Ferreira and Luthria, 2010). Increase in artemisinin content in sun-dried leaves was attributed to improved bioconversion from dihydroartemisinic acid (a precursor of artemisinin) to artemisinin.

Another explanation for the enhanced effects associated with drying is inactivation of deteriorative enzymes such as lipoxygenase and polyphenol oxidases (PPO). PPO, mixtures of monophenol oxidase and catechol oxidase enzymes, are widely present in herbal tissues and can oxidize diphenols in the presence of $\mathrm{O}_{2}$ molecules, causing rapid enzymatic oxidation of natural antioxidants. PPO are reported to be completely inactivated by heating at $80{ }^{\circ} \mathrm{C}$ for $10 \mathrm{~min}$ (Schweiggert et al., 2007). Lim and Murtijaya (2007) suggested that inactivation of PPO may be a reason why dried herbs can have higher TPP and TEAC value than fresh samples.

Cooking of herbs enhances antioxidant activity as a result of the liberation of higher amounts of antioxidants (such as bonded phenolic compounds) resulting from thermal destruction of cell walls and subcellular compartments (Jimenez-Monreal et al., 2009). 
Similarly, cell wall and membrane degradation may be associated with drying and may play an important role in drying-increased TPP and TEAC. In the present study, postharvest drying of herbs enhanced TPP and TEAC in all three herb species with optimal levels obtained with sun- or $40{ }^{\circ} \mathrm{C}$ oven-drying. Regardless of whether bioconversion, enzyme inactivation, or loss of cell wall compartmentalization are the causes, drying can enhance the yield of TPP and TEAC. The dietary intake of sun-dried or low-temperature dried herbs and spices may help increase the bioavailability and absorption of these phytochemical antioxidants. Further studies are underway in our laboratory to evaluate the effects of drying on the bioavailability and bioactivity of these herbs.

For the three herbs evaluated in the current study, high TPP was generally associated with high TEAC. This is in general agreement with previous reports, which often show a linear relationship between TPP and antioxidant capacities. This may be attributed to phenolic compounds having a major contribution to the antioxidant capacity of herbs (Zheng and Wang, 2001).

Solvent extraction of phenolic compounds is widely used in herbs and spices. A number of organic solvents have been used, including water, ethanol, methanol, acetone, and ethyl acetate at different mixture ratios (Areias et al., 2000; Durling et al., 2007). The efficacy of different solvents varies with species and compounds of interest. Lim and Murtijaya (2007) compared methanol and water extractions in freeze-dried sage and found that methanol produced significantly higher phenolic content than water. To extract flavonoids and phenolic acids, Areias et al. (2000) compared in sage the efficacy of petroleum ether, chloroform, ethyl ether, ethyl acetate, acetone, methanol, $30 \%$ ethanol, $80 \%$ ethanol, and boiling water. They found that ethyl ether, ethyl acetate, and acetone were the most efficient for extraction. Areias et al. (2001) also evaluated extraction solvents in fresh peppermint. Comparing petroleum ether, chloroform, ethyl ether, ethyl acetate, acetone, and methanol, they found that ethanol showed the highest efficacy, generating the greatest number of phenolic compounds. The efficacy of ethanol in extraction of fresh herbs is further supported by the results of our current study in which two of the most widely used solvents, methanol and ethanol, were compared. Here we showed that for fresh herbs, ethanol extraction had significantly higher polyphenol contents and TEAC antioxidant capacity, yet for dried herbs, the efficacy of ethanol/methanol extraction varied with different drying treatment. The effectiveness of different solvents varied not only with species and compounds of interest, but with sample drying conditions. Furthermore, solvent choice should include not only yield, but also environmental considerations. Ethanol extraction may be more desirable in some cases because it is less toxic and more environmentally friendly than methanol.

In conclusion, both drying and extraction conditions can significantly impact TPP and antioxidant capacity of peppermint, rosemary, and motherwort leaves. Sun-dried or $40{ }^{\circ} \mathrm{C}$ oven-dried herbs exhibited significantly higher TPP and antioxidant capacity than fresh samples, suggesting low-temperature drying may not only be a good postharvest means to store medicinal/culinary herbs, but provides improved biochemical activity. Exposure to $70^{\circ} \mathrm{C}$ oven-drying caused significant antioxidant loss compared with low-temperature drying. Furthermore, the current study showed that with fresh tissue, ethanol extraction had significantly higher polyphenol contents and TEAC antioxidant capacity than methanol extraction for all three herbs evaluated. Yet for dried herbs, the efficacy of ethanol/methanol extraction varied with different drying treatment, indicating that the efficacy of different solvent extraction varied not only with species and compounds of interest, but with different sample drying conditions as well.

\section{Literature Cited}

Areias, F., P. Valentao, P.B. Andrade, F. Ferreres, and R.M. Seabra. 2000. Flavonoids and phenolic acids of sage: Influence of some agricultural factors. J. Agr. Food Chem. 48:60816084.

Areias, F.M., P. Valentao, P.B. Andrade, F. Ferreres, and R.M. Seabra. 2001. Phenolic fingerprint of peppermint leaves. Food Chem. 73:307311

Berger, M.M. 2005. Can oxidative damage be treated nutritionally? Clin. Nutr. 24:172-183.

Capecka, E., A. Mareczek, and M. Leja. 2005. Antioxidant activity of fresh and dry herbs of some Lamiaceae species. Food Chem. 93:223226.

Dragland, S., H. Senoo, K. Wake, K. Holte, and R. Blomhoff. 2003. Several culinary and medicinal herbs are important sources of dietary antioxidants. J. Nutr. 133:1286-1290.

Durling, N.E., O.J. Catchpole, J.B. Grey, R.F. Webby, K.A. Mitchell, L.Y. Foo, and N.B. Perry. 2007. Extraction of phenolics and essential oil from dried sage (Salvia officinalis) using ethanol-water mixtures. Food Chem. 101:14171424.
Ferreira, J.F.S. and D.L. Luthria. 2010. Drying affects artemisinin, dihydroartemisinic acid, artemisinic acid, and the antioxidant capacity of Artemisia annua L. leaves. J. Agr. Food Chem. 58:1691-1698.

Guan, T.T.Y., S. Cenkowski, and A. Hydamaka. 2005. Effect of drying on the nutraceutical quality of sea buckthorn (Hippophae rhamnoides L. ssp sinensis) leaves. J. Food Sci. 70: E514-E518.

Jimenez-Monreal, A.M., L. Garcia-Diz, M. MartinezTome, M. Mariscal, and M.A. Murcia. 2009. Influence of cooking methods on antioxidant activity of vegetables. J. Food Sci. 74:H97H103.

Kaefer, C.M. and J.A. Milner. 2008. The role of herbs and spices in cancer prevention. J. Nutr. Biochem. 19:347-361.

Knight, J.A. 2000. Review: Free radicals, antioxidants, and the immune system. Ann. Clin. Lab. Sci. 30:145-158.

Konczak, I., D. Zabaras, M. Dunstan, and P. Aguas. 2010. Antioxidant capacity and phenolic compounds in commercially grown native Australian herbs and spices. Food Chem. 122: 260-266.

Lim, Y.Y. and J. Murtijaya. 2007. Antioxidant properties of Phyllanthus amarus extracts as affected by different drying methods. LWTFood Sci. Technol. 40:1664-1669.

Oboh, G. and A.A. Akindahunsi. 2004. Change in the ascorbic acid, total phenol and antioxidant activity of sun-dried commonly consumed green leafy vegetables in Nigeria. Nutr. Health 18:29-36.

Re, R., N. Pellegrini, A. Proteggente, A. Pannala, M. Yang, and C. Rice-Evans. 1999. Antioxidant activity applying an improved ABTS radical cation decolorization assay. Free Radic. Biol. Med. 26:1231-1237.

Schweiggert, U., R. Carle, and A. Schieber. 2007. Conventional and alternative processes for spice production-A review. Trends Food Sci. Technol. 18:260-268.

Singleton, V.L. and J.A. Rossi, Jr. 1965. Colorimetry of total phenolics with phosphomolybdic phosphotungstic acid reagents. Amer. J. Enol. Viticult. 16:144-158.

Tomaino, A., F. Cimino, V. Zimbalatti, V. Venuti, V. Sulfaro, A. De Pasquale, and A. Saija. 2005. Influence of heating on antioxidant activity and the chemical composition of some spice essential oils. Food Chem. 89:549-554.

World Health Organization. 2010. Traditional medicine. World Health Organization (c) 2010. 6 Apr. 2010. $<\mathrm{http}: / / \mathrm{www}$. who.int/mediacentre/factsheets/ fs $134 / \mathrm{en} />$.

Yi, W. and H.Y. Wetzstein. 2010. Biochemical, biological, and histological evaluation of some culinary and medicinal herbs grown under greenhouse and field conditions. J. Sci. Food Agr. 90:1063-1070.

Zheng, W. and S.Y. Wang. 2001. Antioxidant activity and phenolic compounds in selected herbs. J. Agr. Food Chem. 49:5165-5170. 